\title{
Fractional-order Chua's system: discretization, bifurcation and chaos
}

\author{
Ravi P Agarwal ${ }^{1 *}$, Ahmed MA El-Sayed ${ }^{2}$ and Sanaa M Salman ${ }^{3}$
}

${ }^{\text {*Correspondence: }}$

Agarwal@tamuk.edu

1 Department of Mathematics, Texas A and M University, Kingsville, USA

Full list of author information is

available at the end of the article

\begin{abstract}
In this paper we are interested in the fractional-order form of Chua's system. A discretization process will be applied to obtain its discrete version. Fixed points and their asymptotic stability are investigated. Chaotic attractor, bifurcation and chaos for different values of the fractional-order parameter are discussed. We show that the proposed discretization method is different from other discretization methods, such as predictor-corrector and Euler methods, in the sense that our method is an approximation for the right-hand side of the system under study.
\end{abstract}

Keywords: Chua's system; fractional-order differential equations; fixed points; asymptotic stability; chaotic attractor; bifurcation; chaos

\section{Introduction}

In recent years differential equations with fractional order have attracted many researchers' attention because of their applications in many areas of science and engineering; see, for example, [1, 2], and [3]. The need for fractional-order differential equations stems in part from the fact that many phenomena cannot be modeled by differential equations with integer derivatives. The fractional calculus has allowed the operations of integration and differentiation to be applied upon any fractional order. Recently, the theory of fractional differential equations attracted many scientists and mathematicians to work on [4-8]. For stability conditions and synchronization of a system of fractional-order differential equations, one can see [9-11].

We recall the basic definitions (Caputo) and properties of fractional order differentiation and integration.

Definition 1 The fractional integral of order $\beta \in \mathbb{R}^{+}$of the function $f(t), t>0$, is defined by

$$
I^{\beta} f(t)=\int_{0}^{t} \frac{(t-s)^{\beta-1}}{\Gamma(\beta)} f(s) d s,
$$

and the fractional derivative of order $\alpha \in(n-1, n)$ of $f(t), t>0$, is defined by

$$
D^{\alpha} f(t)=I^{n-\alpha} D^{n} f(t), \quad D=\frac{d}{d t} .
$$

In addition, the following results are the main ones in fractional calculus. Let $\beta, \gamma \in \mathbb{R}^{+}$, $\alpha \in(0,1)$,

O2013 Agarwal et al.; licensee Springer. This is an Open Access article distributed under the terms of the Creative Commons Attribution License (http://creativecommons.org/licenses/by/2.0), which permits unrestricted use, distribution, and reproduction in any medium, provided the original work is properly cited. 
- $I_{a}^{\beta}: L^{1} \rightarrow L^{1}$, and if $f(x) \in L^{1}$, then $I_{a}^{\gamma} I_{a}^{\beta} f(x)=I_{a}^{\gamma+\beta} f(x)$.

- $\lim _{\beta \rightarrow n} I_{a}^{\beta} f(x)=I_{a}^{n} f(x)$ uniformly on $[a, b], n=1,2,3, \ldots$, where $I_{a}^{1} f(x)=\int_{a}^{x} f(s) d s$.

- $\lim _{\beta \rightarrow 0} I_{a}^{\beta} f(x)=f(x)$ weakly.

- If $f(x)$ is absolutely continuous on $[a, b]$, then $\lim _{\alpha \rightarrow 1} D_{a}^{\alpha} f(x)=\frac{d f(x)}{d x}$.

To solve fractional-order differential equations, there are two famous methods: frequency domain methods [12] and time domain methods [13]. In recent years it has been shown that the second method is more effective because the first method is not always reliable in detecting chaos [14] and [15].

Often it is not desirable to solve a differential equation analytically, and one turns to numerical or computational methods.

In [16], a numerical method for nonlinear fractional-order differential equations with constant or time-varying delay was devised. It should be noticed that the fractional differential equations tend to lower the dimensionality of the differential equations in question; however, introducing delay in differential equations makes them infinite dimensional. So, even a single ordinary differential equation with delay could display chaos.

Dealing with fractional-order differential equations as dynamical systems is somehow new and has motivated the leading research literature recently; see, for example, $[17,18]$ and [19]. The non-local property of fractional differential equations means that the next state of a system not only depends on its current state but also on its historical states. This property is very close to the real world, and thus fractional differential equations have become popular and have been applied to dynamical systems.

On the other hand, some examples of dynamical systems generated by piecewise constant arguments were studied in [20-24].

\section{Discretization process}

In [25], a discretization process is introduced to discretize the fractional-order differential equations, and we take Riccati's fractional-order differential equations as an example. We noticed that when the fractional-order parameter $\alpha \rightarrow 1$, Euler's discretization method is obtained. In [26], the same discretization method is applied to the logistic fractional-order differential equation. We concluded that Euler's method is able to discretize first-order difference equations; however, we succeeded in discretizing a second-order difference equation.

Here, we are interested in applying the discretization method to a system of differential equations like Chua's system which is one of the autonomous differential equations capable of generating chaotic behavior. This system is well known and has been studied widely.

Let $\alpha \in(0,1)$ and consider the differential equation of fractional order

$$
\begin{aligned}
& D^{\alpha} x(t)=f(x(t)), \quad t>0 . \\
& x(0)=x_{0}, \quad t \leq 0 .
\end{aligned}
$$

The corresponding equation with a piecewise constant argument

$$
D^{\alpha} x(t)=f\left(x\left(r\left[\frac{t}{r}\right]\right)\right), \quad x(t)=x_{0}, t \leq 0 .
$$


Let $t \in[0, r)$, then $\frac{t}{r} \in[0,1)$. So, we get

$$
D^{\alpha} x(t)=f\left(x_{0}\right), \quad t \in[0, r) .
$$

Thus

$$
x_{1}(t)=x_{0}+\frac{t^{\alpha}}{\Gamma(1+\alpha)} f\left(x_{0}\right) .
$$

Let $t \in[r, 2 r)$, then $\frac{t}{r} \in[1,2)$. So, we get

$$
D^{\alpha} x(t)=f\left(x_{1}(r)\right), \quad t \in[r, 2 r)
$$

Thus

$$
x_{2}(t)=x_{1}(r)+\frac{(t-r)^{\alpha}}{\Gamma(1+\alpha)} f\left(x_{1}(r)\right) .
$$

Let $t \in[2 r, 3 r)$, then $\frac{t}{r} \in[2,3)$. So, we get

$$
D^{\alpha} x(t)=f\left(x_{2}(r)\right), \quad t \in[2 r, 3 r) .
$$

Thus

$$
x_{3}(t)=x_{1}(r)+\frac{(t-2 r)^{\alpha}}{\Gamma(1+\alpha)} f\left(x_{2}(r)\right) .
$$

Repeating the process, we get when $t \in[n r,(n+1) r)$, then $\frac{t}{r} \in[n, n+1)$. So, we get

$$
D^{\alpha} x(t)=f\left(x_{n}(n r)\right), \quad t \in[n r,(n+1) r) .
$$

Thus

$$
x_{n+1}(t)=x_{n}(r)+\frac{(t-n r)^{\alpha}}{\Gamma(1+\alpha)} f\left(x_{n}(r)\right) .
$$

Consider Chua's dynamical system with cubic nonlinearity (see $[27,28]$ )

$$
\begin{aligned}
& x=\gamma\left(y+\frac{1}{7} x-\frac{2}{7} x^{3}\right), \\
& y=x-y+z, \\
& z=-\beta y .
\end{aligned}
$$

In [28], the author studied the effect of the fractional dynamics in Chua's system. It has been demonstrated that the usual idea of system order must be modified when fractional derivatives are present. 
Here, we are concerned with fractional-order Chua's system given by

$$
\begin{aligned}
& D^{\alpha} x(t)=\gamma\left(y+\frac{1}{7} x-\frac{2}{7} x^{3}\right), \\
& D^{\alpha} y(t)=x-y+z, \\
& D^{\alpha} z(t)=-\beta y .
\end{aligned}
$$

Actually, we are interested in discretizing fractional-order Chua's system with piecewise constant arguments given in the form

$$
\begin{aligned}
& D^{\alpha} x(t)=\gamma\left(y\left(r\left[\frac{t}{r}\right]\right)+\frac{1}{7} x\left(r\left[\frac{t}{r}\right]\right)-\frac{2}{7} x\left(r\left[\frac{t}{r}\right]\right)^{3}\right), \\
& D^{\alpha} y(t)=x\left(r\left[\frac{t}{r}\right]\right)-y\left(r\left[\frac{t}{r}\right]\right)+z\left(r\left[\frac{t}{r}\right]\right), \\
& D^{\alpha} z(t)=-\beta y\left(r\left[\frac{t}{r}\right]\right)
\end{aligned}
$$

with initial conditions $x(0)=x_{o}, y(0)=y_{o}$, and $z(0)=z_{o}$.

The proposed discretization method has the following steps.

(1) Let $t \in[0, r)$, then $\frac{t}{r} \in[0,1)$. So, we get

$$
\begin{aligned}
& D^{\alpha} x(t)=\gamma\left(y_{0}+\frac{1}{7} x_{0}-\frac{2}{7} x_{0}^{3}\right), \quad t \in[0, r), \\
& D^{\alpha} y(t)=x_{0}-y_{0}+z_{0}, \\
& D^{\alpha} y(t)=-\beta y_{0}
\end{aligned}
$$

and the solution of (7) is given by

$$
\begin{aligned}
x_{1}(t) & =x_{0}+I^{\alpha}\left(\gamma\left(y_{0}+\frac{1}{7} x_{0}-\frac{2}{7} x_{0}^{3}\right)\right) \\
& =x_{0}+\left(\gamma\left(y_{0}+\frac{1}{7} x_{0}-\frac{2}{7} x_{0}^{3}\right)\right) \int_{0}^{t} \frac{(t-s)^{\alpha-1}}{\Gamma(\alpha)} d s \\
& =x_{0}+\left(\gamma\left(y_{0}+\frac{1}{7} x_{0}-\frac{2}{7} x_{0}^{3}\right)\right) \frac{t^{\alpha}}{\Gamma(1+\alpha)}, \\
y_{1}(t) & =y_{0}+I^{\alpha}\left(x_{0}-y_{0}+z_{0}\right) \\
& =y_{0}+\left(x_{0}-y_{0}+z_{0}\right) \int_{0}^{t} \frac{(t-s)^{\alpha-1}}{\Gamma(\alpha)} d s \\
& =y_{0}+\left(x_{0}-y_{0}+z_{0}\right) \frac{t^{\alpha}}{\Gamma(1+\alpha)}, \\
z_{1}(t) & =z_{0}+I^{\alpha}\left(-\beta y_{0}\right) \\
& =z_{0}+\left(-\beta y_{0}\right) \int_{0}^{t} \frac{(t-s)^{\alpha-1}}{\Gamma(\alpha)} d s \\
& =z_{0}+\left(-\beta y_{0}\right) \frac{t^{\alpha}}{\Gamma(1+\alpha)} .
\end{aligned}
$$


(2) Let $t \in[r, 2 r)$, then $\frac{t}{r} \in[1,2)$. So, we get

$$
\begin{aligned}
& D^{\alpha} x(t)=\gamma\left(y_{1}(r)+\frac{1}{7} x_{1}(r)-\frac{2}{7} x_{1}(r)^{3}\right), \quad t \in[r, 2 r) \\
& D^{\alpha} y(t)=x_{1}(r)-y_{1}(r)+z_{1}(r) \\
& D^{\alpha} y(t)=-\beta y_{1}(r)
\end{aligned}
$$

and the solution of (7) is given by

$$
\begin{aligned}
x_{2}(t) & =x_{1}(r)+I^{\alpha}\left(\gamma\left(y_{1}(r)+\frac{1}{7} x_{1}(r)-\frac{2}{7} x_{1}(r)^{3}\right)\right) \\
& =x_{1}(r)+\left(\gamma\left(y_{1}(r)+\frac{1}{7} x_{1}(r)-\frac{2}{7} x_{1}(r)^{3}\right)\right) \int_{0}^{t} \frac{(t-s)^{\alpha-1}}{\Gamma(\alpha)} d s \\
& =x_{1}(r)+\left(\gamma\left(y_{1}(r)+\frac{1}{7} x_{1}(r)-\frac{2}{7} x_{1}(r)^{3}\right)\right) \frac{t^{\alpha}}{\Gamma(1+\alpha)}, \\
y_{2}(t) & =y_{1}(r)+I^{\alpha}\left(x_{1}(r)-y_{1}(r)+z_{1}(r)\right) \\
& =y_{1}(r)+\left(x_{1}(r)-y_{1}(r)+z_{1}(r)\right) \int_{0}^{t} \frac{(t-s)^{\alpha-1}}{\Gamma(\alpha)} d s \\
& =y_{1}(r)+\left(x_{1}(r)-y_{1}(r)+z_{1}(r)\right) \frac{t^{\alpha}}{\Gamma(1+\alpha)}, \\
z_{2}(t) & =z_{1}(r)+I^{\alpha}\left(-\beta y_{1}(r)\right) \\
& =z_{1}(r)+\left(-\beta y_{1}(r)\right) \int_{0}^{t} \frac{(t-s)^{\alpha-1}}{\Gamma(\alpha)} d s \\
& =z_{1}(r)+\left(-\beta y_{1}(r)\right) \frac{t^{\alpha}}{\Gamma(1+\alpha)} .
\end{aligned}
$$

Repeating the process, we can easily deduce that the solution of (7) is given by

$$
\begin{aligned}
& x_{n+1}(t)=x_{n}(n r)+\frac{(t-n r)^{\alpha}}{\Gamma(1+\alpha)}\left(\gamma\left(y_{n}(n r)+\frac{1}{7} x_{n}(n r)-\frac{2}{7} x_{n}(n r)^{3}\right)\right), \quad t \in[n r,(n+1) r), \\
& y_{n+1}(t)=y_{n}(n r)+\frac{(t-n r)^{\alpha}}{\Gamma(1+\alpha)}\left(x_{n}(n r)-y_{n}(n r)+z_{n}(n r)\right), \\
& z_{n+1}(t)=z_{n}(n r)+\frac{(t-n r)^{\alpha}}{\Gamma(1+\alpha)}\left(-\beta y_{n}(n r)\right) .
\end{aligned}
$$

Let $t \rightarrow(n+1) r$, we obtain the discretization

$$
\begin{aligned}
& x_{n+1}((n+1) r)=x_{n}(n r)+\frac{r^{\alpha}}{\Gamma(1+\alpha)}\left(\gamma\left(y_{n}(n r)+\frac{1}{7} x_{n}(n r)-\frac{2}{7} x_{n}(n r)^{3}\right)\right), \\
& y_{n+1}((n+1) r)=y_{n}(n r)+\frac{r^{\alpha}}{\Gamma(1+\alpha)}\left(x_{n}(n r)-y_{n}(n r)+z_{n}(n r)\right), \\
& z_{n+1}((n+1) r)=z_{n}(n r)+\frac{r^{\alpha}}{\Gamma(1+\alpha)}\left(-\beta y_{n}(n r)\right),
\end{aligned}
$$


which can be rewritten as

$$
\begin{aligned}
& x_{n+1}=x_{n}+\frac{r^{\alpha}}{\Gamma(1+\alpha)}\left(\gamma\left(y_{n}+\frac{1}{7} x_{n}-\frac{2}{7} x_{n}^{3}\right)\right) \\
& y_{n+1}=y_{n}+\frac{r^{\alpha}}{\Gamma(1+\alpha)}\left(x_{n}-y_{n}+z_{n}\right), \\
& z_{n+1}=z_{n}+\frac{r^{\alpha}}{\Gamma(1+\alpha)}\left(-\beta y_{n}\right) .
\end{aligned}
$$

Remark 1 It should be noticed that if $\alpha \rightarrow 1$ in (8), we deduce the Euler discretization method of Chua's system [29].

It is worth to mention here that many discretization methods, such as Euler's method and predictor-corrector method, have been applied to Chua's system (4). Euler's method discretization is an approximation for the derivative while the predictor-corrector method is an approximation for the integral. However, our proposed discretization method here is an approximation for the right-hand side as it is pretty clear from formula (8).

\section{Fixed points and their asymptotic stability}

Now we study the asymptotic stability of the fixed points of system (8) which has three fixed points:

- $f\left(x_{1}=(0,0,0)\right.$,

- $f\left(x_{2}=\left(\frac{1}{\sqrt{2}}, 0, \frac{-1}{\sqrt{2}}\right)\right.$,

- $f\left(x_{3}=\left(\frac{-1}{\sqrt{2}}, 0, \frac{1}{\sqrt{2}}\right)\right.$.

By considering a Jacobian matrix for one of these fixed points and calculating their eigenvalues, we can investigate the stability of each fixed point based on the roots of the system characteristic equation [30].

Linearizing system (8) about $f x_{1}$ yields the following characteristic equation:

$$
\begin{aligned}
P(\lambda)= & \lambda^{3}+\left(s\left(1-\frac{1}{7} \gamma\right)-3\right) \lambda^{2}+\left(3-2 s\left(1-\frac{1}{7} \gamma\right)-s^{2}\left(\beta+\frac{8}{7} \gamma\right)\right) \lambda \\
& +\left(-1+s\left(1-\frac{1}{7} \gamma\right)+s^{2}\left(\beta+\frac{8}{7} \gamma\right)+\frac{1}{7} \beta \gamma s^{3}\right)=0,
\end{aligned}
$$

where $s=\frac{r^{\alpha}}{\Gamma(1+\alpha)}$. Let

$$
\begin{aligned}
& a_{1}=s\left(1-\frac{1}{7} \gamma\right)-3, \\
& a_{2}=3-2 s\left(1-\frac{1}{7} \gamma\right)-s^{2}\left(\beta+\frac{8}{7} \gamma\right), \\
& a_{3}=-1+s\left(1-\frac{1}{7} \gamma\right)+s^{2}\left(\beta+\frac{8}{7} \gamma\right)+\frac{1}{7} \beta \gamma s^{3} .
\end{aligned}
$$

Now, let $a_{1}=A, a_{2}=B$, and $a_{3}=C$. From the Jury test, if $P(1)>0, P(-1)<0$, and $a_{3}<1$, $\left|b_{3}\right|>b_{1}, c_{3}>\left|c_{2}\right|$, where $b_{3}=1-a_{3}^{2}, b_{2}=a_{1}-a_{3} a_{2}, b_{1}=a_{2}-a_{3} a_{1}, c_{3}=b_{3}^{2}-b_{1}^{2}$, and $c_{2}=$ $b_{3} b_{2}-b_{1} b_{2}$, then the roots of $P(\lambda)$ satisfy $\lambda<1$ and thus $f\left(x_{1}\right.$ is asymptotically stable. This is not satisfied here since $\gamma$ and $\beta$ are positive and so $\left|b_{3}\right|<b_{1}$. That is, $f i x_{1}$ is unstable. 


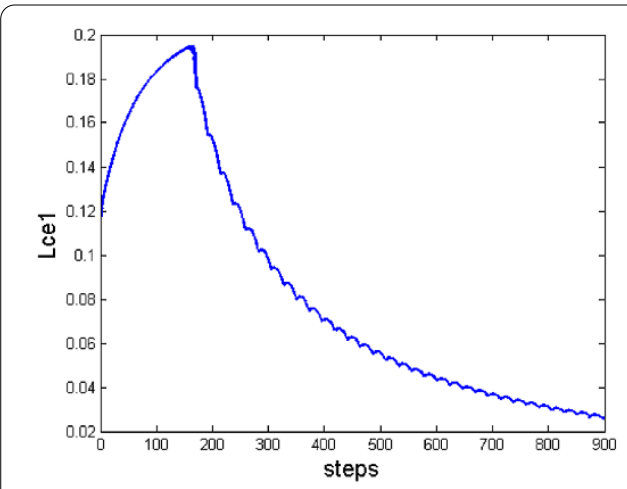

(a)

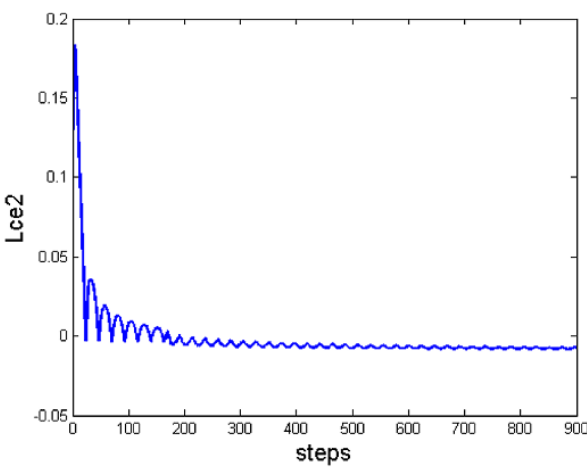

(b)

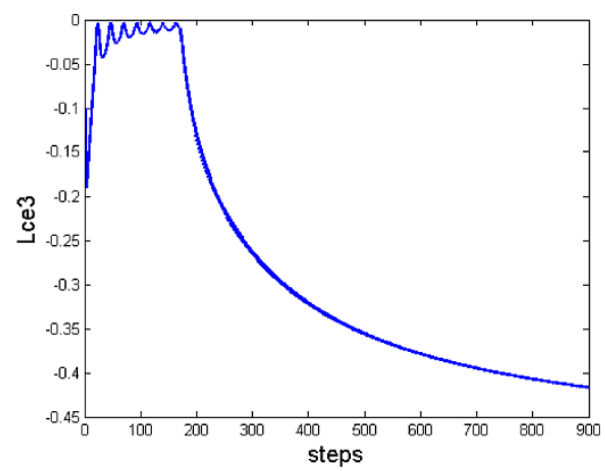

(c)

Figure 1 Lyapunov characteristic exponents (LCEs) for system (8).

While linearizing system (8) about $f i x_{2}$ or $f i x_{3}$ yields the following characteristic equation:

$$
\begin{aligned}
F(\lambda)= & \lambda^{3}+\left(s\left(1+\frac{2}{7} \gamma\right)-3\right) \lambda^{2}+\left(3-s\left(2+\frac{4}{7} \gamma\right)-s^{2}\left(\beta+\gamma-\frac{2}{7} \gamma\right)\right) \lambda \\
& +\left(-1+s\left(1+\frac{2}{7} \gamma\right)+s^{2}\left(\beta+\gamma-\frac{2}{7} \gamma\right)-\frac{2}{7} \gamma \beta s^{3}\right)=0 .
\end{aligned}
$$

We let $a_{11}=s\left(1+\frac{2}{7} \gamma\right)-3, a_{22}=3-s\left(2+\frac{4}{7} \gamma\right)-s^{2}\left(\beta+\gamma-\frac{2}{7} \gamma\right)$, and $a_{33}=-1+s\left(1+\frac{2}{7} \gamma\right)+$ $s^{2}\left(\beta+\gamma-\frac{2}{7} \gamma\right)-\frac{2}{7} \gamma \beta s^{3}$. From the Jury test, if $F(1)>0, F(-1)<0$, and $a_{33}<1,\left|b_{33}\right|>b_{11}$, $c_{33}>\left|c_{22}\right|$, where $b_{33}=1-a_{33}^{2}, b_{22}=a_{11}-a_{33} a_{22}, b_{11}=a_{22}-a_{33} a_{11}, c_{33}=b_{33}^{2}-b_{11}^{2}$, and $c_{22}=$ $b_{33} b_{22}-b_{11} b_{22}$, then the roots of $F(\lambda)$ satisfy $\lambda<1$ and thus $f i x_{2}$ or $f i x_{3}$ is asymptotically stable. We can check easily that $F(1)<0$, that is, both $f\left(x_{2}\right.$ and $f i x_{3}$ are unstable.

\section{Attractors, bifurcation and chaos}

Since the Lyapunov exponent is a good indicator for existence of chaos, we compute the Lyapunov characteristic exponents (LCEs) via the householder QR based methods described in [31]. LCEs play a key role in the study of nonlinear dynamical systems and they are the measure of sensitivity of solutions of a given dynamical system to small changes in the initial conditions. One feature of chaos is the sensitive dependence on initial conditions; for a chaotic dynamical system, at least one LCE must be positive. Since for non- 
Figure 2 Strange attractor of (8) with $\alpha=0.75, \beta$

Figure 2 Strange attractor
$=16, r=0.01, \gamma=29.5$

Figure 3 Attractor of (8) with $\alpha=0.85, \beta=16, r$ $=0.01, \gamma=29.5$.

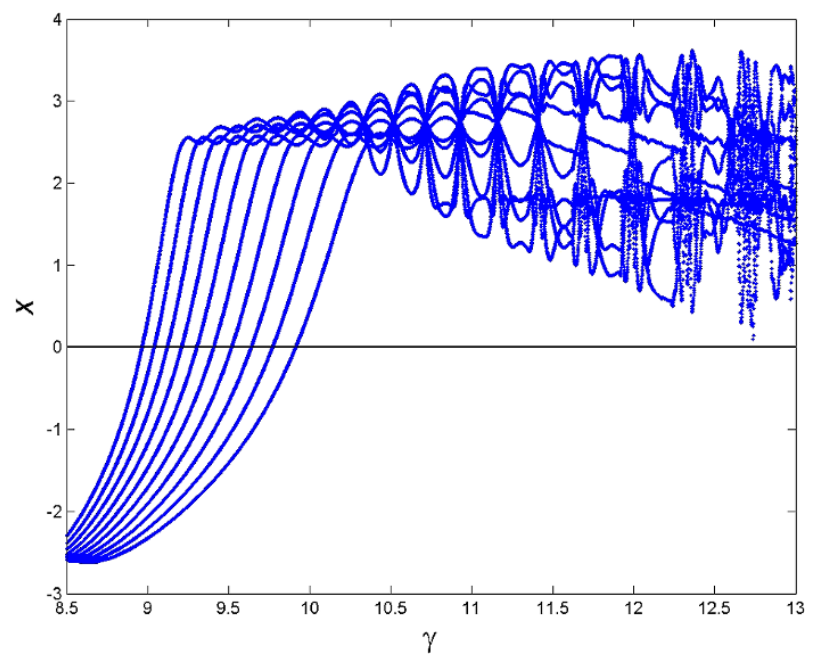

Figure 4 Bifurcation diagram of (8) with $\alpha=0.75, \beta=16, r=0.01$.

chaotic systems all LCEs are non-positive, the presence of a positive LCE has often been used to help determine if a system is chaotic or not. We find that LCE1 $=0.0263$, LCE2 $=$ -0.0077 , and LCE3 $=-0.4160$. Figure 1 shows the LCEs for system (8) for parameter values $r=0.0105, \beta=\frac{100}{7}$, and $\alpha=0.75$ with initial conditions $\left(x_{0}, y_{0}, z_{0}\right)=(0.2,0.4,0.2)$.

On the other hand, we show some attractors of system (8) for different $\alpha$. The numerical experiments show that playing with the parameter $\alpha$ away from $\alpha=0.75$ will not produce 


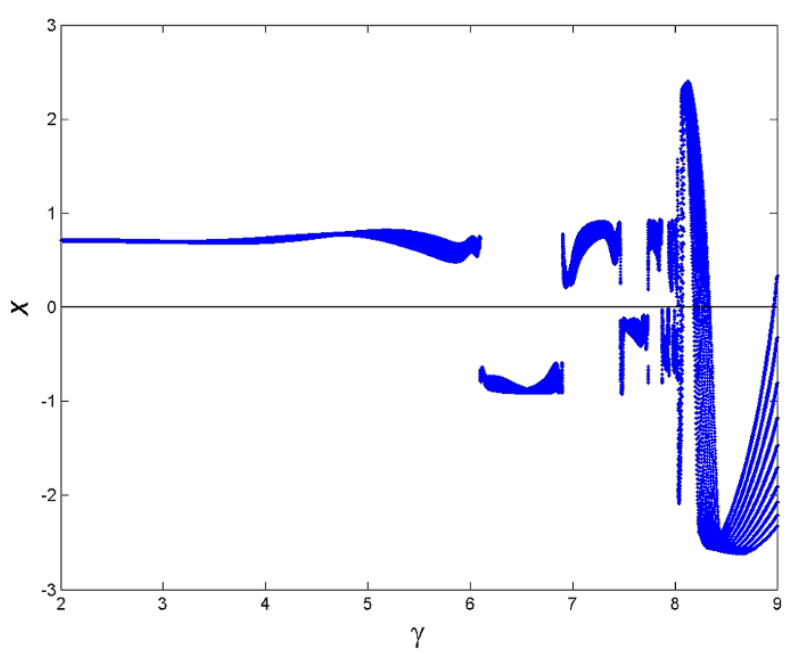

Figure 5 Bifurcation diagram of (8) with $\alpha=0.75, \beta=16, r=0.01$.

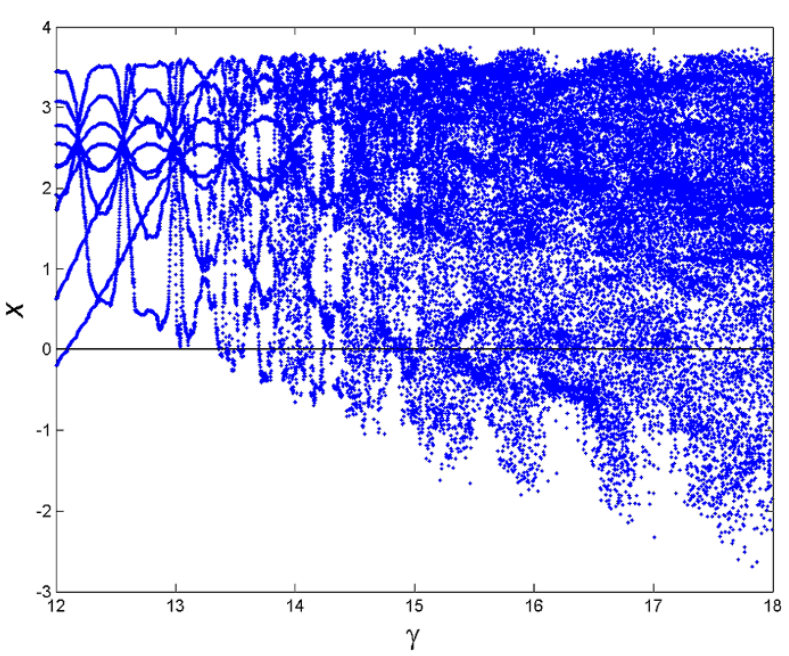

Figure 6 Bifurcation diagram of (8) with $\alpha=0.75, \beta=\frac{100}{7}, r=0.0105$.

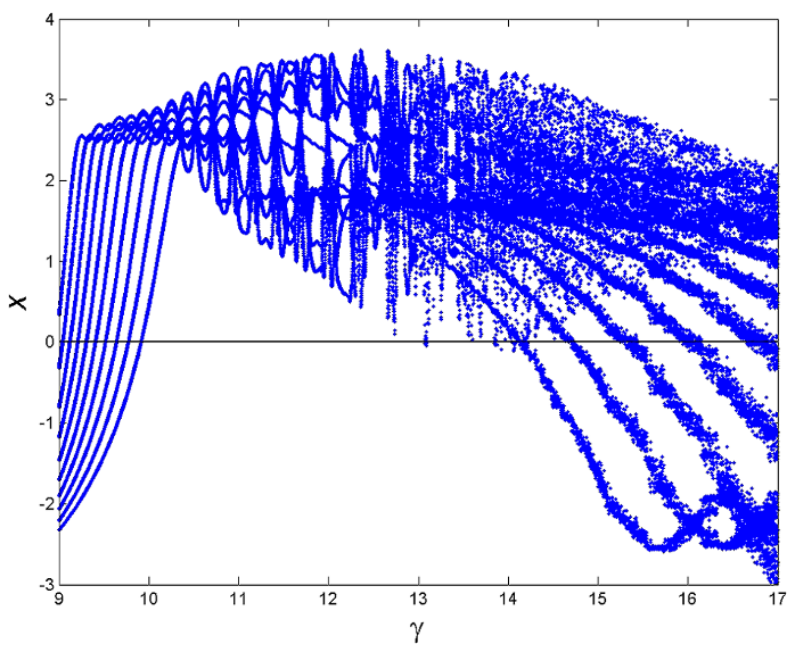

Figure 7 Bifurcation diagram of (8) with $\alpha=0.75, \beta=16, r=0.01$. 


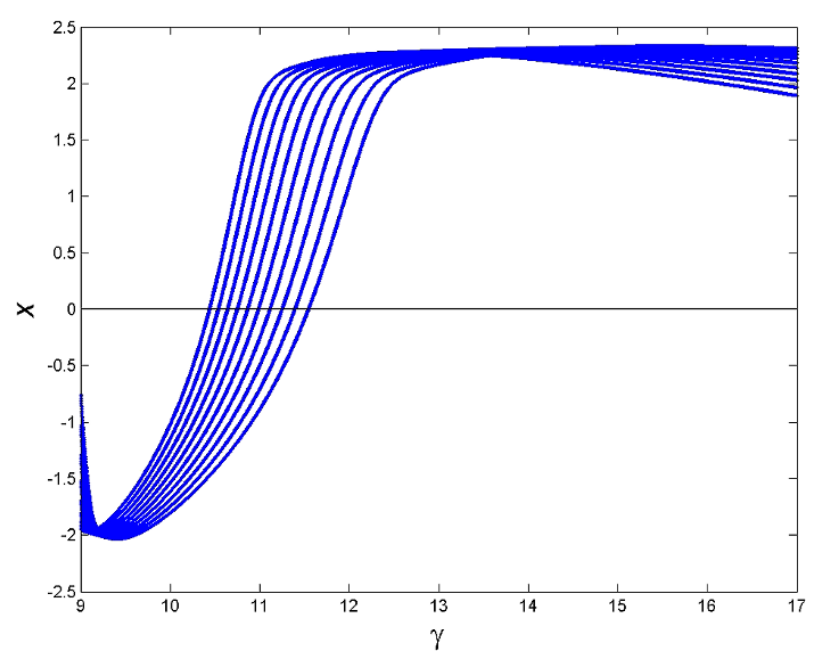

Figure 8 Bifurcation diagram of (8) with $\alpha=0.85, \beta=16, r=0.01$.

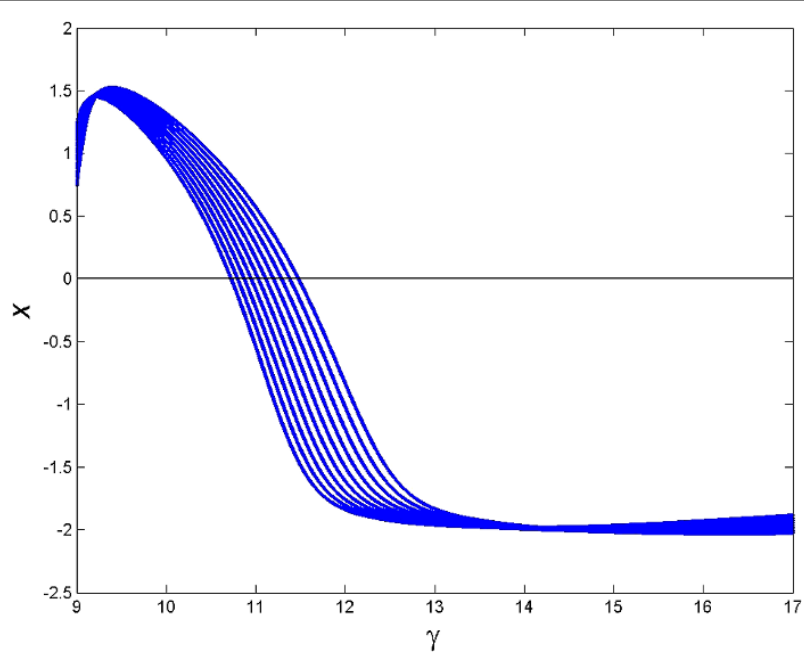

Figure 9 Bifurcation diagram of (8) with $\alpha=0.95, \beta=16, r=0.01$.

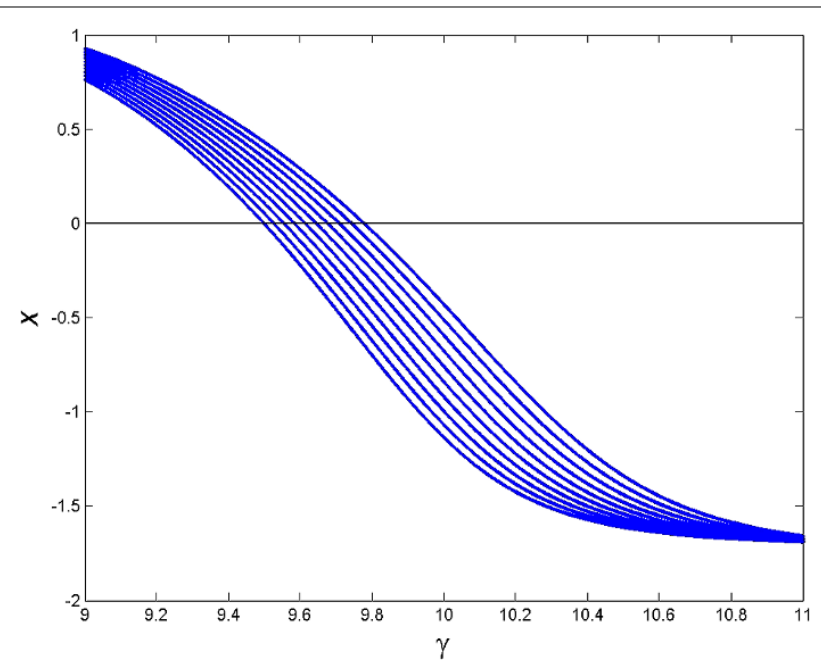

Figure 10 Bifurcation diagram of (8) with $\alpha=1, \beta=16, r=0.01$. 
Agarwal et al. Advances in Difference Equations 2013, 2013:320

Page 11 of 13

http://www.advancesindifferenceequations.com/content/2013/1/320

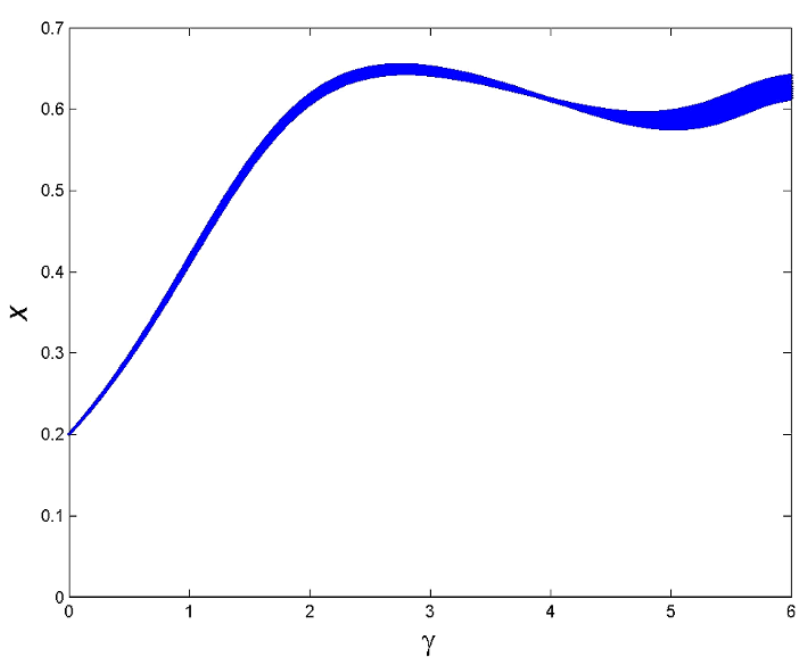

Figure 11 Bifurcation diagram of (8) with $\alpha=1, \beta=16, r=0.01$.

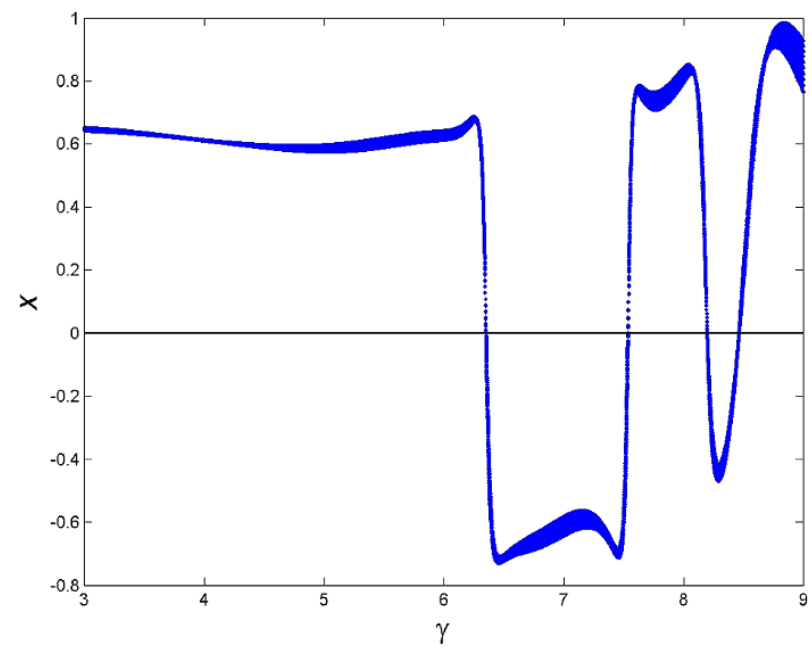

Figure 12 Bifurcation diagram of (8) with $\alpha=1, \beta=16, r=0.01$.

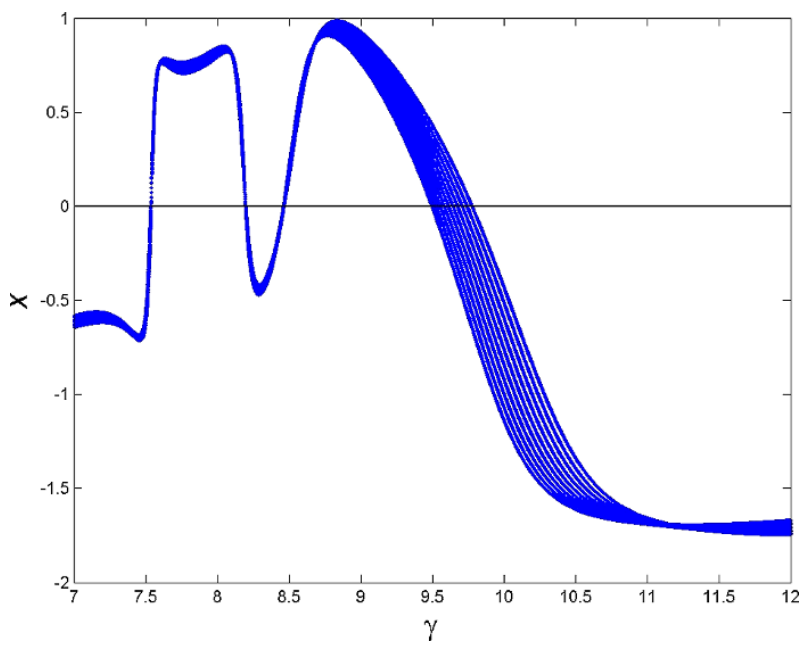

Figure 13 Bifurcation diagram of (8) with $\alpha=0.1, \beta=16, r=0.01$. 
any bifurcation diagrams. Figures 2 and 3 show attractors of system (8), while Figures 4-13 show bifurcation diagrams for the same system.

\section{Conclusion}

A discretization method is introduced to discretize fractional-order differential equations and we take Chua's system with cubic nonlinearity for our purpose. We have noticed that when $\alpha \rightarrow 1$, the discretization will be Euler's discretization [29]. In addition, we carried out the numerical simulation when $\alpha \rightarrow 1$, we did not get any bifurcation at all. Actually, this is not surprising since we did the same in Rössler's system in its discrete version. When we contacted Prof. Dr. Rössler himself about why we were not getting any bifurcation diagrams, he assured our results. Finally, it is not clear in this situation why the parameter $\alpha$ takes one value only to produce bifurcation and chaos diagrams.

On the other hand, we show some attractors of system (8) for different $\alpha$. The numerical experiments show that playing with the parameter $\alpha$ away from $\alpha=0.75$ will not produce any bifurcation diagrams.

Competing interests

The authors declare that they have no competing interests.

\section{Authors' contributions}

The authors declare that the study was realized in collaboration with the same responsibility. All authors read and approved the final manuscript.

\section{Author details}

'Department of Mathematics, Texas A and M University, Kingsville, USA. ²Faculty of Science, Alexandria University, Alexandria, Egypt. ${ }^{3}$ Faculty of Education, Alexandria University, Alexandria, Egypt.

\section{Acknowledgements}

The authors would like to thank the referees of this manuscript for their valuable comments and suggestions.

Received: 10 May 2013 Accepted: 4 October 2013 Published: 14 Nov 2013

\section{References}

1. Bhalekara, S, Daftardar-Gejjib, V, Baleanuc, D, Magine, R: Transient chaos in fractional Bloch equations. Comput. Math. Appl. 64, 3367-3376 (2012)

2. Faieghi, M, Kuntanapreeda, S, Delavari, H, Baleanu, D: LMI-based stabilization of a class of fractional-order chaotic systems. Nonlinear Dyn. 72, 301-309 (2013)

3. Golmankhaneh, AK, Arefi, R, Baleanu, D: The proposed modified Liu system with fractional order. Adv. Math. Phys. 2013, 186037 (2013)

4. Das, S: Functional Fractional Calculus for System Identification and Controls. Springer, Berlin (2007)

5. El-Sayed, AMA, El-Mesiry, A, El-Saka, H: On the fractional-order logistic equation. Appl. Math. Lett. 20, 817-823 (2007)

6. El-Sayed, AMA: On the fractional differential equations. J. Appl. Math. Comput. 49(2-3), 205-213 (1992)

7. El-Sayed, AMA: Nonlinear functional-differential equations of arbitrary orders. Nonlinear Anal. 33, 181-186 (1998)

8. Podlubny, I: Fractional Differential Equations. Academic Press, London (1999)

9. Matouk, AE: Stability conditions, hyperchaos and control in a novel fractional order hyperchaotic system. Phys. Lett. A 373, 2166-2173 (2009)

10. Matouk, AE: Chaos synchronization between two different fractional systems of Lorenz family. Math. Probl. Eng. 2009 Article ID 572724 (2009)

11. Matouk, AE: On some stability conditions and hyperchaos synchronization in the new fractional order hyperchaotic Chen system. In: Proceedings of the 3rd International Conference ICCSA, LeHavre-Normandy, France, June 29-July 02 (2009)

12. Sun, $H$, Abdelwahed, A, Onaral, B: Linear approximation for transfer function with a pole of fractional order. IEEE Trans Autom. Control 29, 441-444 (1984)

13. Diethelm, K, Ford, NJ, Freed, AD: A predictor-corrector approach for the numerical solution of fractional differential equations. Nonlinear Dyn. 29, 3-22 (2002)

14. Tavazoei, MS, Haeri, M: Unreliability of frequency domain approximation in recognizing chaos in fractional order systems. IET Signal Process. 1, 171-181 (2007)

15. Tavazoei, MS, Haeri, M: Limitation of frequency domain approximation for detecting chaos in fractional order systems. Nonlinear Anal., Theory Methods Appl. 69, 1299-1320 (2008)

16. Wang, Z: A numerical method for delayed fractional-order differential equations. J. Appl. Math. 2013, 256071 (2013)

17. Erjaee, GH: On analytical justification of phase synchronization in different chaotic systems. Chaos Solitons Fractals 3 , 1195-1202 (2009) 
18. Yan, JP, Li, CP: On chaos synchronization of fractional differential equations. Chaos Solitons Fractals 32, 725-735 (2007)

19. Wu, GC, Baleanu, D: Discrete fractional logistic map and its chaos. Nonlinear Dyn. (2013). doi:10.1007/s11071-013-1065-7

20. Akhmet, MU: Stability of differential equations with piecewise constant arguments of generalized type. Nonlinear Anal. 68, 794-803 (2008)

21. Akhmet, MU, Altntana, D, Ergenc, T: Chaos of the logistic equation with piecewise constant arguments. arXiv:1006.4753 (2010)

22. El-Sayed, AMA, Salman, SM: Chaos and bifurcation of discontinuous dynamical systems with piecewise constant arguments. Malaya J. Mat. 1, 14-18 (2012)

23. El-Sayed, AMA, Salman, SM: Chaos and bifurcation of the logistic discontinuous dynamical systems with piecewise constant arguments. Malaya J. Mat. 3, 14-20 (2013)

24. El-Sayed, AMA, Salman, SM: The unified system between Lorenz and Chen systems: a discretization process. Electron. J. Math. Anal. Appl. 1, 318-325 (2013)

25. El-Sayed, AMA, Salman, SM: On a discretization process of fractional order Riccati's differential equation. J. Fract. Calc. Appl. 4, 251-259 (2013)

26. El-Sayed, AMA, Salman, SM: On a discretization process of fractional-order Logistic differential equation. J. Egypt. Math. Soc. (accepted)

27. Stegemann, C, Albuquerque, HA, Rech, PC: Some two dimensional parameter space of a Chua system with cubic nonlinearity. Chaos, Interdiscip. J. Nonlinear Sci. 20, 817-823 (2007)

28. Hartely, TT, Lorenzo, CF, Qammer, HK: Chaos in a fractional order Chau's system. IEEE Trans. Circuits Syst. I, Fundam. Theory Appl. 42, 485-490 (1995)

29. Elaidy, SN: An Introduction to Difference Equations, 3rd edn. Undergraduate Texts in Mathematics. Springer, New York (2005)

30. Holmgren, R: A First Course in Discrete Dynamical Systems. Springer, New York (1994)

31. Udwadia, FE, von Bremen, $\mathrm{H}$ : A note on the computation of the largest $p$-Lyapunov characteristic exponents for nonlinear dynamical systems. J. Appl. Math. Comput. 114, 205-214 (2000)

10.1186/1687-1847-2013-320

Cite this article as: Agarwal et al.: Fractional-order Chua's system: discretization, bifurcation and chaos. Advances in Difference Equations 2013, 2013:320

\section{Submit your manuscript to a SpringerOpen ${ }^{\circ}$ journal and benefit from:}

- Convenient online submission

- Rigorous peer review

- Immediate publication on acceptance

- Open access: articles freely available online

- High visibility within the field

- Retaining the copyright to your article 